Gilles Rousselier, ${ }^{1}$ Jean-Claude Devaux, ${ }^{2}$ Gérard Mottet,${ }^{2}$ and

Georges Devesa ${ }^{1}$

\section{A Methodology for Ductile Fracture Analysis Based on Damage Mechanics: An Illustration of a Local Approach of Fracture}

REFERENCE: Rousselier, G., Devaux, J.-C., Mottet, G., and Devesa, G., "A Methodology for Ductile Fracture Analysis Based on Damage Mechanics: An Illustration of a Local Approach of Fracture," Nonlinear Fracture Mechanics: Volume II-Elastic-Plastic Fracture, ASTM STP 995, J. D. Landes, A. Saxena, and J. G. Merkle, Eds., American Society for Testing and Materials, Philadelphia, 1989, pp. 332-354

\begin{abstract}
Major solutions needed in fracture analysis are $(a)$ simple and accurate material characterization and $(b)$ easy transferring of material data to cracked structures. In the proposed methodology, constitutive relationships, including cavity growth and coalescence, are used. Material characterization is based on the simple notched tension test. Several structural steels have been characterized, especially A508 steel, and the direct transferring of material data has been demonstrated with tests on circumferentially cracked tension specimens.

In addition, the extrapolation of material data to different inclusion contents and temperatures was attempted, with favorable results for the first factor through a specific parameter of the model. The temperature dependence of A508 steel ductility is related to an inverse strain rate effect on the flow curve; the modeling of this effect gives encouraging results, but it must be refined to produce an effective prediction.
\end{abstract}

KEY WORDS: ductile fracture, damage mechanics, local approach to fracture, crack initiation, stable crack growth, A508 steel, inclusion contents, notched tension test, fracture mechanics, nonlinear fracture mechanics

Some of the major problems engineers have to cope with in fracture analysis are the following:

(a) material characterization, that is, the generation of adequate data from specimen testing, and

(b) the transferring of fracture mechanics data to the structural analysis of components.

As a matter of fact, the generation of material data can be money and time consuming: for example, the determination of $J-\Delta a$ resistance curves is still a toilsome task, though some progress was gained with partial unloading compliance methods. Frequently, existing material data do not correspond to the specific application (in reference to temperature, strain rate, irradiation, aging, and so forth), and hazardous extrapolations are necessary.

'Senior engineer and engineer, respectively, Electricité de France, Service Réacteurs Nucléaires et Echangeurs, Les Renardières, 77250 Moret-sur-Loing, France

${ }^{2}$ Senior engineer and engineer, respectively, Framatome, Centre de Calcul de la Division des Fabrications, BP 13, 71380 Saint-Marcel, France.
With permission of ASTM

(March 11, 2019)

in Landes, J., Saxena, A., Merkle, J., Eds., Nonlinear

Fracture Mechanics:

Volume II, Elastic-Plastic

Fracture, STP995V2-EB,

ASTM International, West

Conshohocken, PA, 1988.

https://doi.org/10.1520./

STP995V2-EB. 
On the other hand, the transferring of fracture mechanics data to industrial components may be questionable, especially if complex situations are involved, including heterogeneous materials, residual stresses, thermomechanical loadings, historical effects, or other factors.

A local approach of fracture is particularly suited to solving these two difficulties. It can be defined very generally as a combination of the following:

(a) the computation of local stress and strain in the most loaded parts of a component or a structure and

(b) physical fracture models corresponding to various mechanisms: cleavage, ductile fracture, creep, and so forth.

Actually, the generation of material data is simple in a local approach to fracture. The application of a local approach to fracture in complex structural situations is direct, as is shown in the accompanying paper by Devaux et al. [1], and the progress of numerical analysis software and hardware makes the computational cost a less and less significant consideration.

The present paper deals only with ductile fracture. The development of a new methodology for ductile fracture analysis was undertaken a few years ago in a cooperative program between Electricité de France and Framatome; this methodology is based on a local approach to fracture and damage mechanics. The paper focuses on material characterization, deriving from the very simple notched tension test $(a)$ the ductile fracture model and parameters calibration and $(b)$ the prediction of the inclusion content and temperature effects on ductile fracture properties. In addition, the ability of the model to predict crack initiation and growth in a structure is briefly presented in the last section of this paper.

\section{The Ductile Fracture Model-Parameters Calibration}

Ductile fracture results from the formation, growth, and coalescence of cavities. The case of intergranular cavities, which corresponds more specifically to creep damage, is not considered here.

In a local approach to ductile fracture, a damage variable is introduced. This variable is computed at every point on the structure. Its evolution is a function of local stresses and strains. For example, if isotropic cavity growth only is considered, the damage variable can be the equivalent cavity radius $R$; its evolution can be given by the well-known equation by Rice and Tracey

$$
\frac{\dot{R}}{R}=0.283 \dot{\epsilon}_{\mathrm{eq}}^{p} \exp \left(\frac{3 \sigma_{m}}{2 \sigma_{\mathrm{eq}}}\right)
$$

where $\sigma_{m}=\sigma_{i i} / 3$ is the mean hydrostatic stress, and $\sigma_{\mathrm{eq}}$ and $\dot{\boldsymbol{\epsilon}}_{\mathrm{eq}}^{p}$ are the equivalent stress and plastic strain rate, respectively. Then, a very simple criterion for cavity coalescence can be formulated: it is based on the assumption of a critical cavity growth $\left(R / R_{0}\right)_{c}$, where $R_{0}$ is the initial cavity radius. This criterion has been widely used in local approach to fracture $[1,2]$.

In the proposed methodology a somewhat different formulation is used. The model refers to the basic assumptions of "continuum damage mechanics," that is, the damage variable is included in the constitutive relationships of the material; there is no conceptual difference between the hardening variable related to plastic deformation and the damage variable.

The constitutive relationships are derived from a plastic potential $F$, and yield criterion 
$F=0$, with the normality rule $[3,4]$. The function $F$ is

$$
F(\tilde{\boldsymbol{\sigma}}, p, \beta)=\tilde{\boldsymbol{\sigma}}_{\text {eq }}-R(p)+B(\beta) D \exp \left(\frac{\tilde{\boldsymbol{\sigma}}_{m}}{\sigma_{1}}\right)
$$

where $D$ and $\sigma_{1}$ are constants, $p$ is the hardening variable (the cumulated plastic strain if $\dot{p}=\dot{\boldsymbol{\epsilon}}_{\mathrm{eq}}^{p}$ ), and $\beta$ is the damage variable. $\beta$ is defined in the Appendix by $\dot{\beta}=\dot{v} / v$, where $v$ is the average volume of the cavities. Both $p$ and $\beta$ are scalar, as isotropic hardening and damage are assumed. The function $R(p)$ defines the hardening curve of the material (stressstrain curve). The function $B(\beta)$ is

$$
B(\beta)=\frac{\sigma_{1} f_{0} \exp \beta}{1-f_{0}+f_{0} \exp \beta}=\sigma_{1} f
$$

where $f_{0}$ is a constant, indicating the initial volume fraction of cavities, and $f$ is the actual volume fraction of cavities.

The derivation of Eqs 2 and 3 is detailed in the Appendix; it is not significant to the following results. What has to be remembered is that damage results in softening of the material and fracture proceeds from the competition between hardening and damage: when damage overcomes the hardening of the material at the tip of a crack or in the center of a tension specimen, there is strain localization, which rapidly results in tremendous strains and damage. The stresses decrease abruptly and vanish, and the zone of strain and damage localization can be assimilated to a crack.

Therefore, it is not necessary to introduce a critical value of the damage variable. As a matter of fact, fracture can occur for different values of the damage variable, depending on the previous history of the material. The only material parameters are the constants of Eqs 2 and 3: $D, \sigma_{1}$, and $f_{0}$. When steep gradients of stress and strain exist, as at the tip of a crack, it is acknowledged that a critical distance $\ell_{c}$ has to be added to the local fracture criterion: $\ell_{c}$ reflects the statistical aspects of fracture, the interaction between the inclusions and the crack tip in the case of ductile fracture.

From cavity growth measurements and theoretical considerations, the authors concluded that the constant $D$ does not depend on the material and can be taken to be equal to 2 , at least for the initial volume fraction of cavities $f_{0}$ equal to or smaller than $10^{-3}[4]$. The three remaining material parameters to be calibrated are these:

(a) $f_{0}$, related to the volume fraction of inclusions $f_{v}$;

(b) $\sigma_{1}$, related to the resistance of the metal matrix to the growth and coalescence of cavities; and

(c) $\ell_{c}$, related to interinclusion spacing.

\section{Calibration Procedure}

How can the parameters $f_{0}, \sigma_{1}$, and $\ell_{c}$ be calibrated? Good estimates of $f_{0}$ and $\ell_{c}$ are obtained from metallographic examinations of the inclusions [4]

$$
\begin{gathered}
f_{0}=f_{v} \frac{\left(d_{x} d_{y}\right)^{1 / 2}}{d_{z}} \\
\ell_{c}=\frac{5}{\left(N_{v}\right)^{1 / 3}}
\end{gathered}
$$


where $d_{x}, d_{y}$, and $d_{z}$ are the average dimensions of the inclusions, $z$ is the direction of the load, and $N_{v}$ the number of inclusions per unit of volume. The value $f_{v}$ can be calculated either from the metallographic examinations or from the chemical analysis (Franklin's formula [5]).

The stress level of the hardening curve of the material gives a poor estimate of $\sigma_{1}[4]$. In practice, mechanical testing is necessary to calibrate $\sigma_{1}$. The basic specimens are axisymmetric notched tension specimens. The use of these specimens might seem surprising as far as fracture mechanics is concerned, but the following must be noted:

1. In a local approach to fracture, uncracked geometries as well as cracked geometries can be analyzed, and material parameters can be transferred from one geometry to the other.

2. Once a crack has been initiated in the center of a notched tension specimen, it responds like a cracked specimen.

The calibration procedure is depicted in Fig. 1. In notched tension specimens, the initiation of a crack results in a marked change of the slope of the load-displacement curve, Point A in Fig. 1, as shown by tests interrupted before and after this point [6]. In the numerical simulation of the test, the location of this point depends on the parameters $\sigma_{1}$ and $f_{0}$ only, and not on $\ell_{c}$, as there is initially no steep gradients of stress and strain. If $f_{0}$ is known, the comparison of experimental and numerical curves makes it possible to calibrate the parameter $\sigma_{1}$.

The crack propagation rate, which depends on $\ell_{c}$, is related to the slope of the postinitiation curve, $\mathrm{AB}$ in Fig. 1. In a numerical calculation $\ell_{c}$ is the length of the finite elements in front of the crack tip. So, the estimated $\ell_{c} \simeq 5\left(N_{v}\right)^{-1 / 3}$ can be checked with the comparison of numerical and experimental postinitiation curves.

Let us point out that no crack growth measurements are necessary, which makes the calibration procedure a very simple one compared with $J-\Delta a$ resistance curve testing. On the other hand, a numerical simulation of the tests is required, though, fortunately, existing

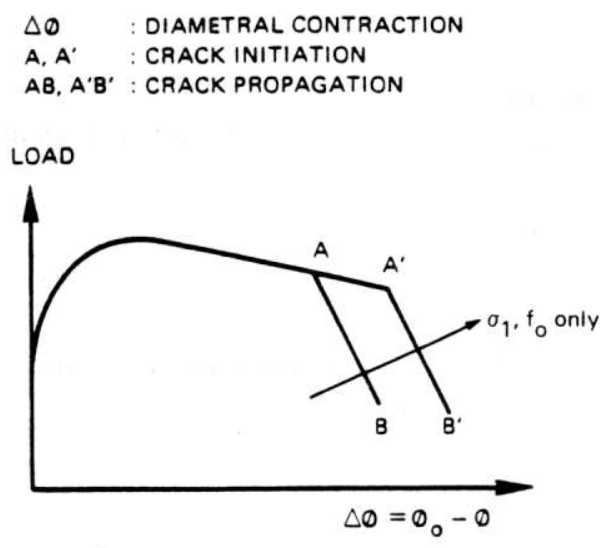

(a) EFFECT OF $\sigma_{1}$ (OR $f_{0}$ )

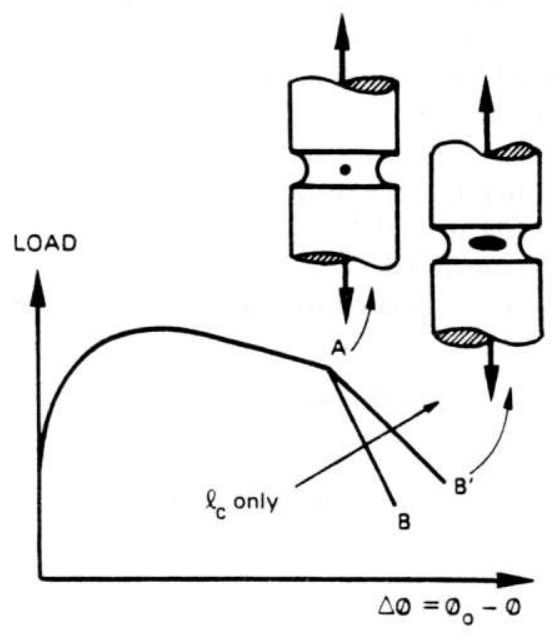

(b) EFFECT OF $\ell_{c}$

FIG. 1-Numerical load-displacement curves of a notched tension specimen (schematic). 
calculations can often be used. Anyway, two-dimensional elastoplastic analyses are no longer a problem with recent computers. The use of axisymmetric specimens makes these twodimensional analyses perfectly suited.

The constitutive relationships with ductile fracture damage were implemented in two finiteelement programs:

(a) the TITUS program, a general system for mechanical analysis developed by Framatome; and

(b) the ALIBABA program, a two-dimensional program developed by Electricité de France.

Numerous cross-checks of the two programs demonstrated that both give practically identical results. So the results obtained with the two programs will not be differentiated in the following sections. With both, the large changes in geometry are taken into account by modifying the coordinates at each load step.

\section{Ductile Fracture Characterization of Structural Steels}

The foregoing calibration procedure was applied to several structural steels:

1. A low-alloy rotor steel-The specimens were taken in the tangential direction of a 500-mm-thick turbine disk.

2. An austenitic weld-The specimens were taken at the bottom of the 70 -mm-thick weld in the transverse direction.

3. A first heat, Heat A subsequently, of A508 Class 3 steel-The specimens were taken from a nozzle dropout of a 200 -mm-thick pressurized water reactor (PWR) vessel shell, in the long (that is tangential) direction.

The chemical composition of these steels is given in Table 1. The metallographic examinations resulted in $f_{0}=10^{-4}, \ell_{c} \simeq 0.4 \mathrm{~mm}$ for the rotor steel [4] and the austenitic weld [7], and $f_{0}=1.6 \times 10^{-4}, \ell_{c} \simeq 0.55 \mathrm{~mm}$ for Heat A of the A508 steel [8].

The diameter of the minimum cross section of the axisymmetric notched tension specimens was $\phi_{0}=10 \mathrm{~mm}$, and the outer diameter was $18 \mathrm{~mm}$. These specimens only differed in the notch radius, which was $2,4,5$, and $10 \mathrm{~mm}$ for the AE2, AE4, AE5, and AE10 specimens, respectively.

For the rotor steel, two AE5 specimens were tested at $40^{\circ} \mathrm{C}$. The right location of the initiation point was obtained with $\sigma_{1}=490 \mathrm{MPa}$. The experimental and numerical load displacement curves are compared in Fig. 2. The agreement is excellent, and thus the calibration of the parameters is satisfactory. In Fig. 3, the highly damaged zone $(\beta>4.5$, that is, the void growth ratio $v / v_{0}>90$ ), which is assimilated into the cracked zone, is localized in the minimum cross section of the specimen. Its radius is in good agreement with the experimental cracks derived from tests interrupted at the same diametral contraction $\Delta \phi=1.5 \mathrm{~mm}$. The numerical crack resistance curve (loading parameter versus crack area) is shown in Fig. 4; it is very close to a straight line. This feature, which is also observed with other steels, makes it possible to determine the crack resistance curve of a material with a single specimen: the load-displacement curve gives the initiation point, and the interrupted test gives the final crack area.

For the austenitic weld, AE2, AE4, and AE10 specimens were tested at $20^{\circ} \mathrm{C}$ [7]. The minimum cross sections of the specimens were located in the middle of the weld line. The strong anisotropy of the weld metal made the diametral contraction measurements hazard- 


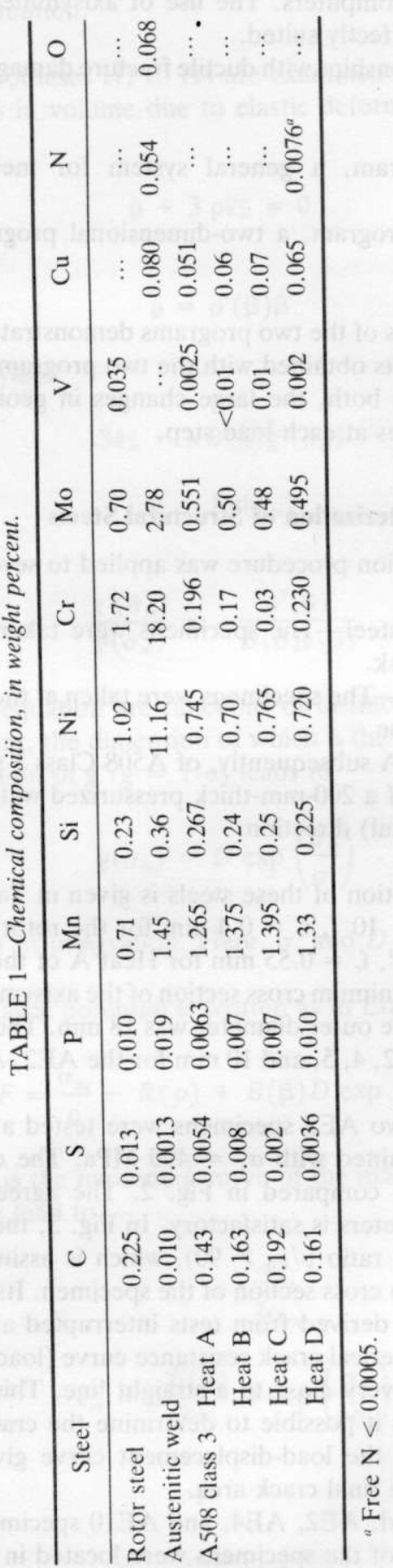




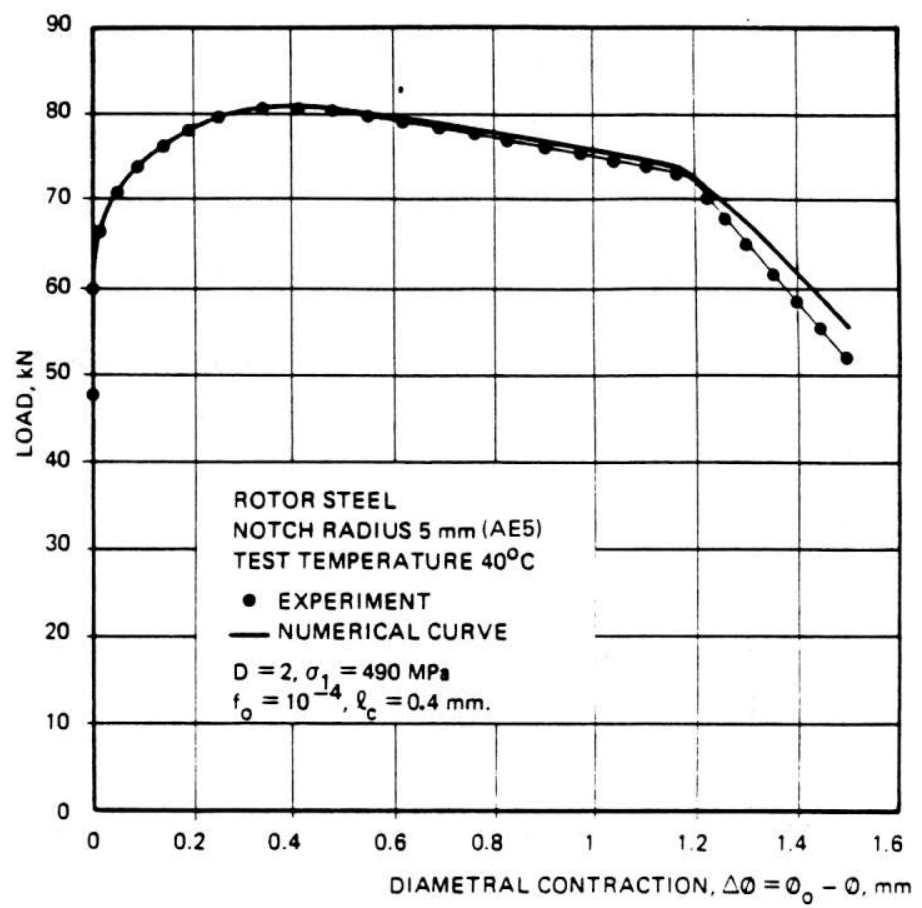

FIG. 2-Load-displacement curve of a rotor steel notched tension specimen.

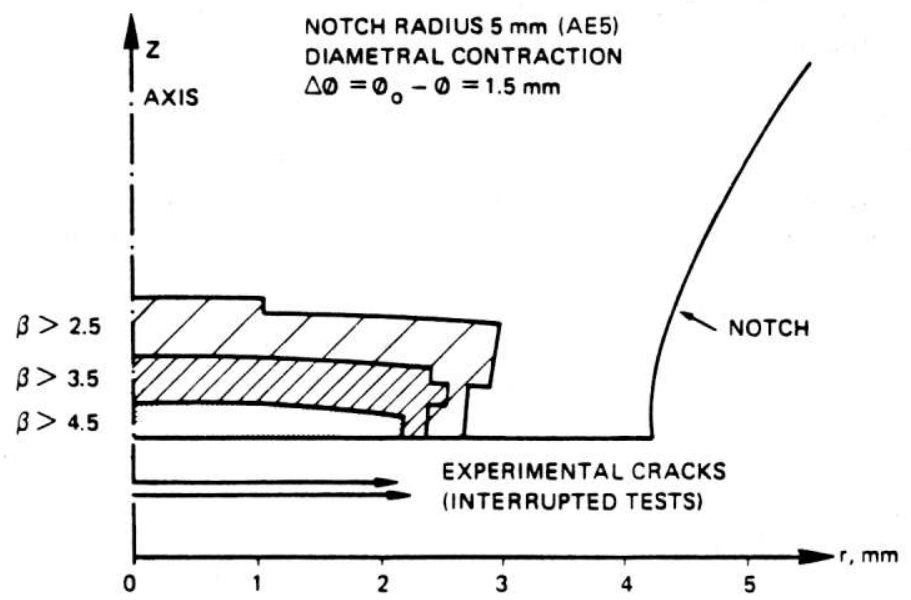

FIG. 3-Damaged zones in a rotor steel notched tension specimen. 


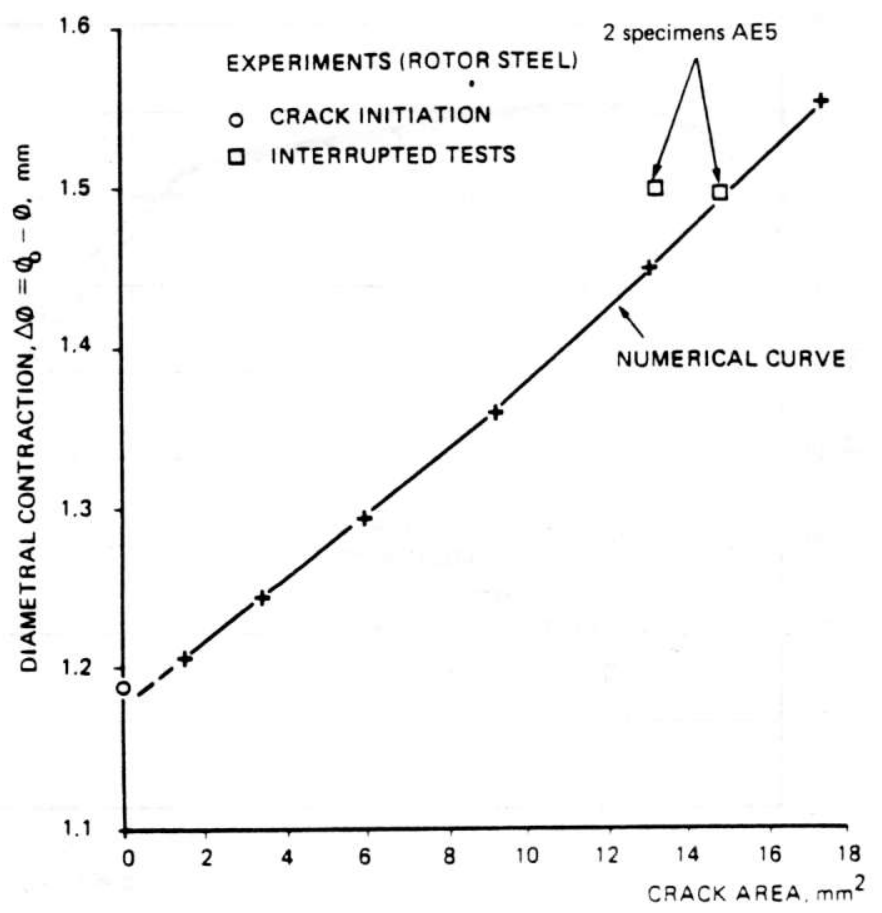

FIG. 4-Crack resistance curve of a rotor steel notched tension specimen.

ous. As a matter of fact, only the diameter at fracture of the specimens, coinciding with crack initiation for this material, could be measured on the broken test pieces. A reasonable agreement between these measurements and the numerical initiation points of the three specimens is obtained with $\sigma_{1}=565 \mathrm{MPa}$ (see Fig. 5). The very steep postinitiation curve calculated for the AE2 specimen is coherent with the experimental coincidence of initiation and fracture (taking into account that the stiffness of the testing machine is finite, which promotes instability). Nevertheless, stable crack growth measurements would be useful to check the value $\ell_{c}=0.4 \mathrm{~mm}$.

For A508 steel, Heat A, two AE2 specimens were tested at $100^{\circ} \mathrm{C}$. The numerical curve with the calibrated value $\sigma_{1}=445 \mathrm{MPa}$ is compared in Fig. 6 with the experimental curves. The calibration of the parameters is satisfactory. In Fig. 7, the localization of damage and the corresponding collapse of longitudinal stress in the vicinity of the minimum cross section of the specimen is shown. (The diametral contraction $\Delta \phi$ is about 2.6 times the end displacement $u_{z}$; crack initiation is at $u_{z} \approx 0.61 \mathrm{~mm}$.)

In the conclusion of this section the following can be asserted:

1. The material characterization can be performed on various steels with simple measurements and tests (some additional specimen testing would be necessary to confirm the characterization of the austenitic weld).

2. The numerical simulation of the notched tension test is in good agreement with the experimental results, for both crack initiation and propagation. 


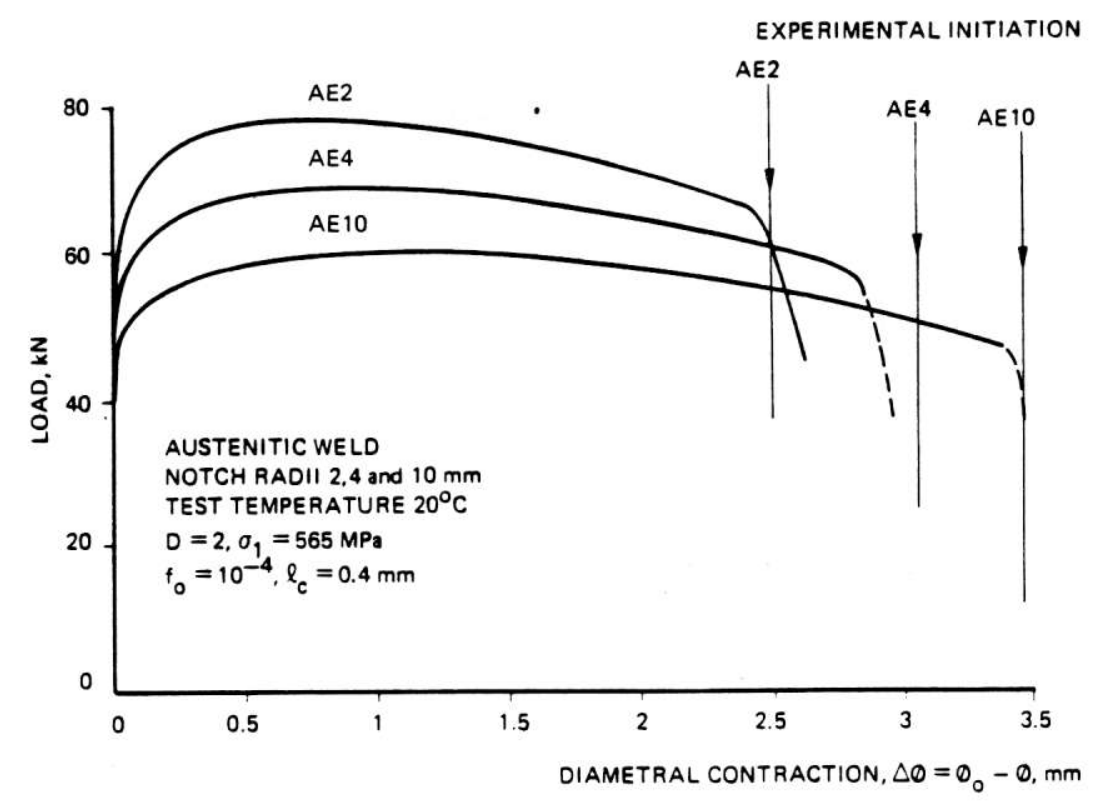

FIG. 5-Numerical load-displacement curves and experimental initiation points of the austenitic weld.

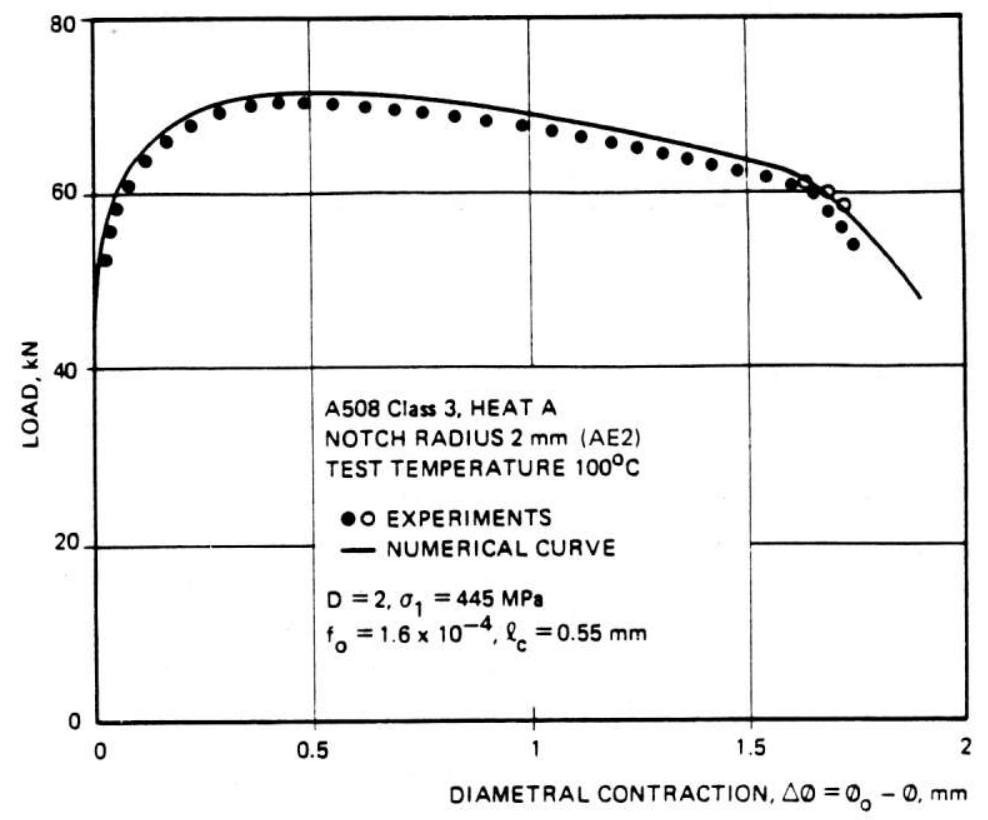

FIG. 6-Load-displacement curve of A508 steel notched tension specimen. 


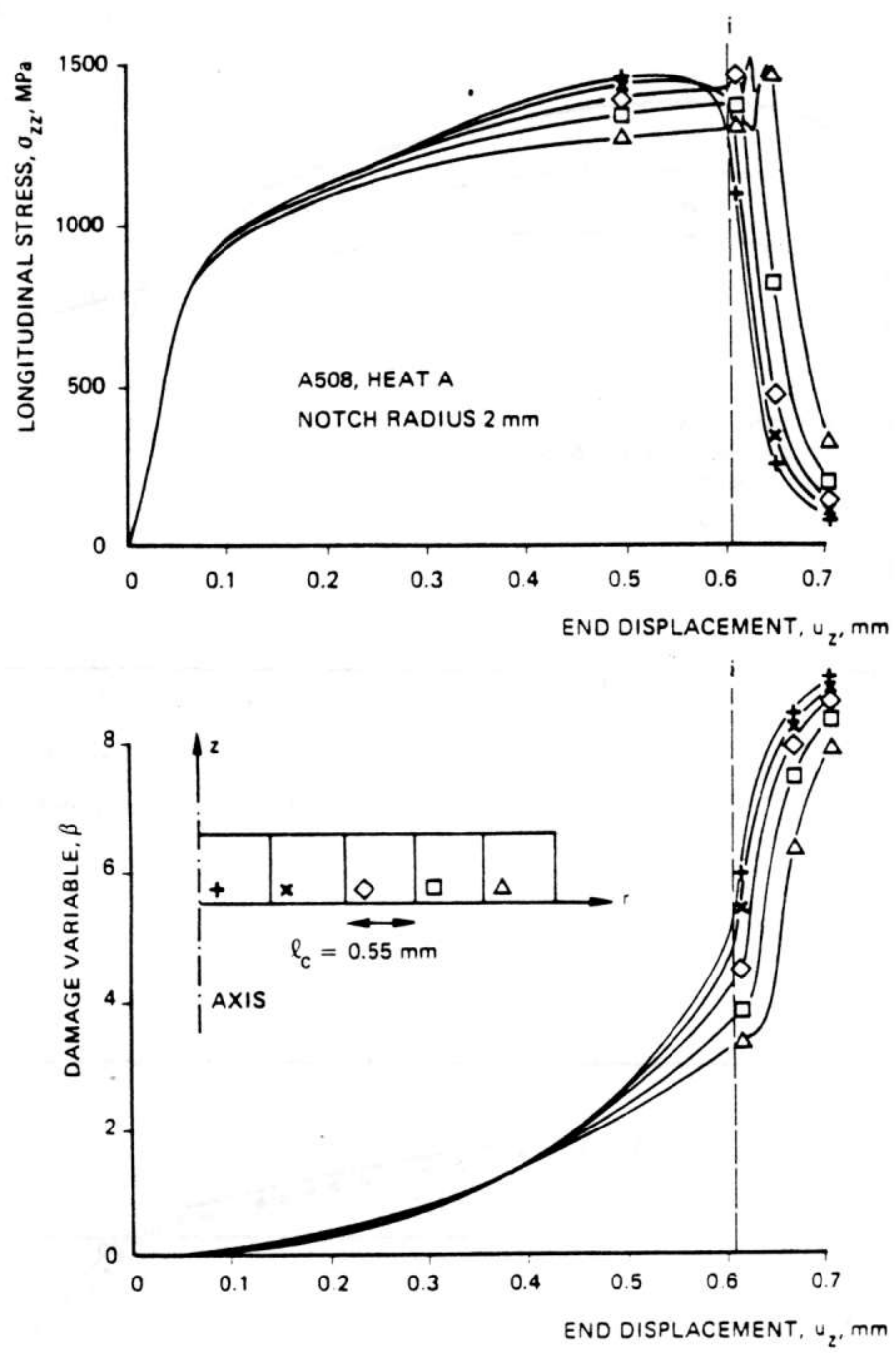

FIG. 7-Longitudinal stress and damage at gauss integration points in the vicinity of the minimum cross section.

\section{Prediction of the Inclusion Content Effect on Ductile Fracture}

In this section two additional heats of A508 Class 3 steel were tested, Heats B and C. For Heat B, the specimens were taken from a nozzle dropout of a PWR vessel shell, in the transverse (that is, axial) direction. For Heat $\mathrm{C}$, the specimens were taken in the longitudinal direction from a 200 -mm-thick cylindrical forging, used for large-scale pressurized thermal shock testing at the Staatliche Materialprüfungsanstalt (MPA) in Stuttgart [9]. The chemical compositions of both steels are given in Table 1. From the metallographic examination of Heat B, a good estimate of the initial volume fraction of cavities to be used in the calculations is $f_{0} \simeq 10^{-3}$ [8]. No metallographic analysis of Heat $C$ has been performed yet, but in this very clean steel the inclusions are expected to be more or less spherical, so that $f_{0} \simeq f_{v}$ can 
be assumed. According to Franklin's formula [5], the volume fraction of inclusions is $f_{v}=$ $7 \times 10^{-5}$.

Considering that the key difference between the three heats of A508 steel is their inclusion content, the point of most interest is whether or not it is possible, with the sole parameter $f_{0}$, to estimate the ductility of the two "unknown" heats, B and C. That is why the first finite-element analyses were performed with the mechanical properties of Heat A (same stress-strain curve, $\sigma_{1}, l^{\prime}$, and other factors) but with different $f_{11}\left[10^{-3}, 1.6 \times 10^{-4}\right.$ (as in Figs. 6 and 7), and $7 \times 10^{-5}$.

The results are given in Fig. 8, together with the mean experimental fracture initiation points deduced from the experiments on AE2 notched specimens. We can conclude that:

1. The parameter $f_{11}$ represents with a good accuracy the effect of inclusion content in the range of interest $\left(10^{-3}\right.$ to $\left.10^{-4}\right)$.

2. As first estimates, $f_{01}=10^{-3}$ and $7 \times 10^{-5}$ can be retained for Heats $\mathrm{B}$ and $\mathrm{C}$.

Note that from Fig. 8 an approximation formula can be proposed between $f_{10}$ and the numerical ductility at fracture initiation $\epsilon_{F}=2 \ln \left(\phi_{1 /} / \phi_{F}\right)$

$$
\boldsymbol{\epsilon}_{F}=0.104 f_{11}^{-0.14}
$$

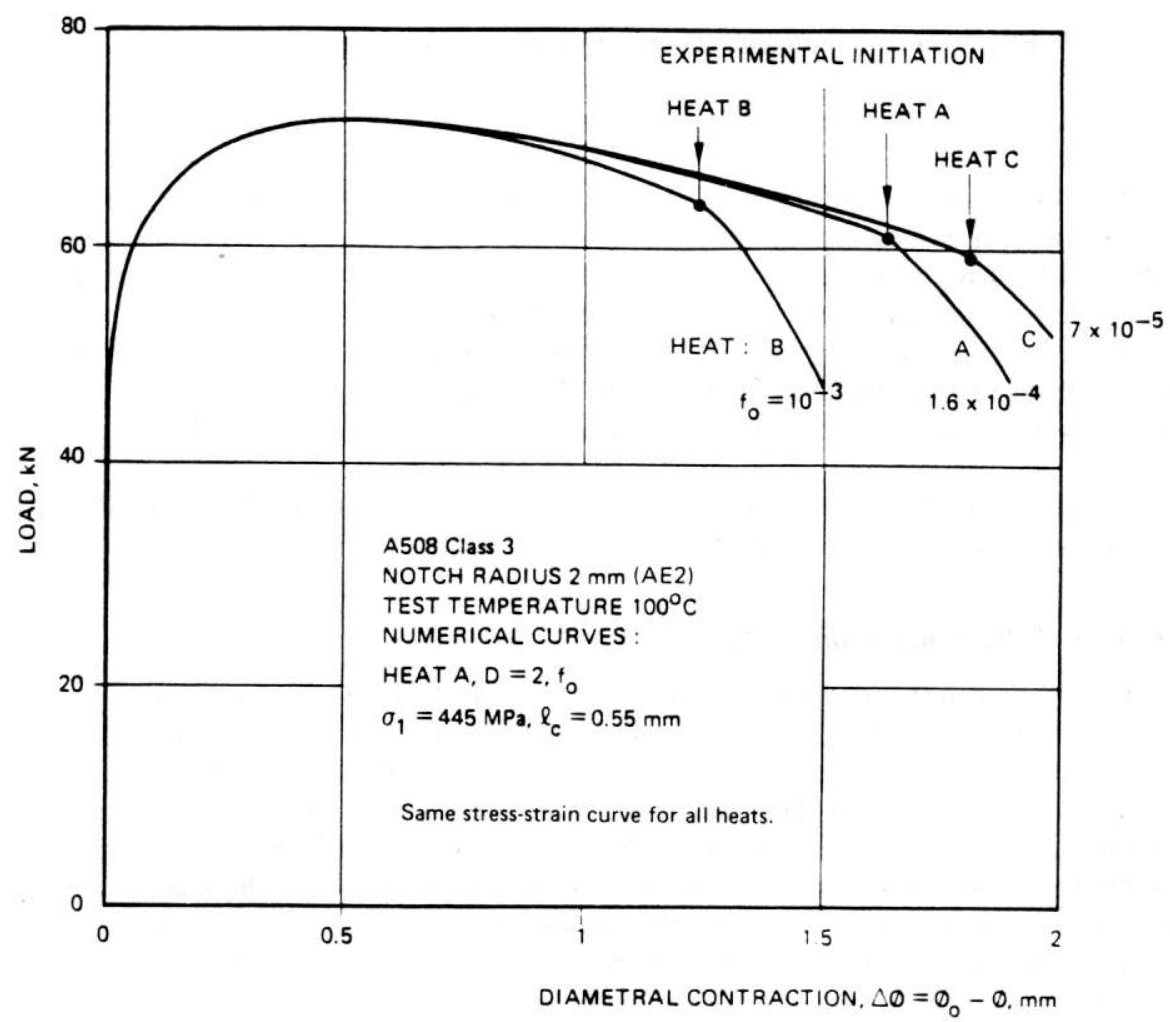

FIG. 8-Numerical load-displacement curves and experimental initiation points of A508 steel. 


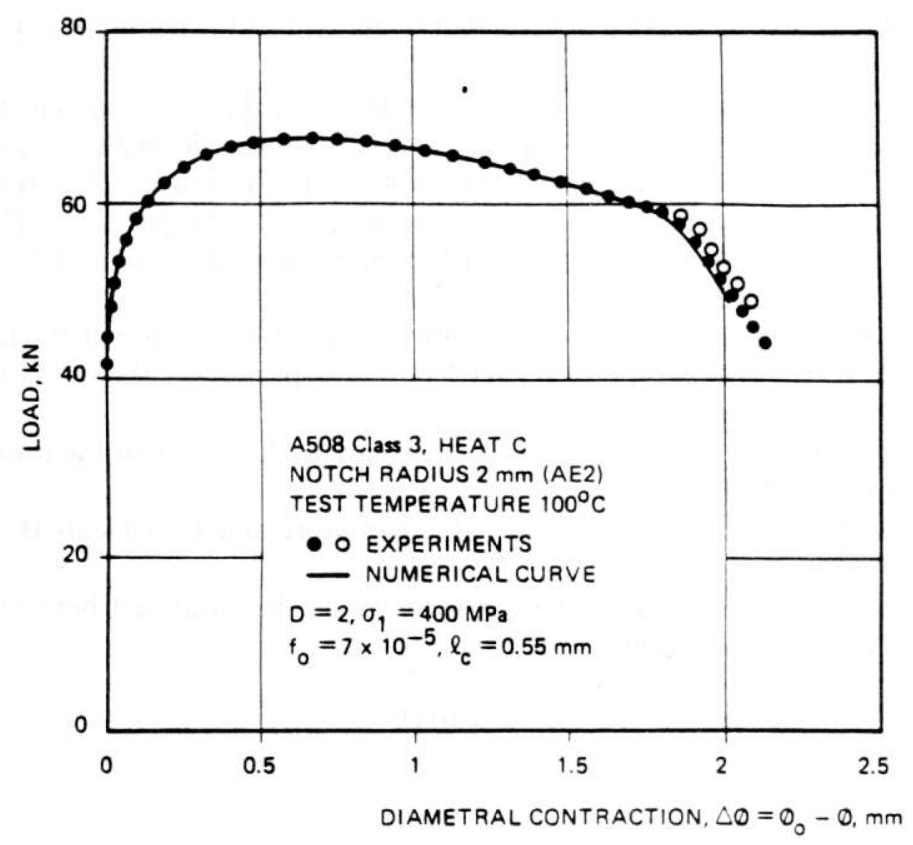

FIG. 9-Load-displacement curve of A508 steel notched tension specimen.

where the three points corresponding to $f_{0}=7 \times 10^{-5}, 1.6 \times 10^{-4}$, and $10^{-3}$ are in per: alignment in a log-log diagram.

Additional calculations were performed with the true stress/strain curves of Heats B C. The stress-strain curves of Heats A and B are very close, so it was not necessary calibrate the parameter $\sigma_{1}\left(\sigma_{1}=445 \mathrm{MPa}\right)$ again. The stress-strain curve of Heat $\mathrm{C}$ significantly lower than those of Heats $\mathrm{A}$ and $\mathrm{B}$, so the parameter $\sigma_{1}$ had to be reduced relation for Heat $\mathrm{C}$. The resulting numerical curve, with $\sigma_{1}=400 \mathrm{MPa}$, is plotted Fig. 9.

In conclusion, a good prediction of the effect of inclusion content on the ductile fract properties of a steel is obtained in the proposed methodology, through the parameter $f_{0}$.

\section{Prediction of the Temperature Effect on Ductile Fracture}

Carbon steels can present a drop in ductility and fracture toughness in the temperature range 100 to $400^{\circ} \mathrm{C}$, known as the "blue brittleness." Earlier work [10] has shown that A508 Class 3 steel presents this phenomenon; it has been demonstrated that the temperature of minimum fracture toughness depends on the strain rate and that there is a clear correlation between this phenomenon and dynamic strain aging, resulting from dynamic interactions between dislocations and intersticial atoms such as nitrogen $[11,12]$. The macroscopic manifestations of dynamic strain aging are the serrated flow observed on smooth tension specimens (Portevin-Le Chatelier effect) and an inverse effect of strain rate on the flow curve (hardening curve) of the material. This inverse effect promotes flow localization between cavities, that is, cavity coalescence, which explains the lower ductility and fracture toughness.

Amar and Pineau have performed tests in the temperature range 100 to $450^{\circ} \mathrm{C}$ on notched 
tension specimens, taken in the longitudinal direction from a nozzle dropout of a PWR vessel shell [12]. The chemical composition of the steel (A508, Heat D) is given in Table 1. Mean deformation in the minimum cross section and diametral contraction at fracture initiation are plotted in Fig. 10. In terms of a local approach to fracture, the authors used the critical cavity growth criterion $\left(R / R_{0}\right)_{c}$ and, of course, the same temperature dependence of $\left(R / R_{0}\right)_{c}$ as in Fig. 10.

An equivalent approach can be performed with the proposed methodology. The parameters $f_{0}$ and $\ell_{c}$, related to the inclusions, should not depend on the temperature. The dynamic strain aging effect, resulting in an easier flow localization in the metal matrix between cavities, is naturally expressed through the parameter $\sigma_{1}$, which is related to the resistance of the metal matrix to the growth and coalescence of cavities. The calibration procedure presented in the first section can be used, and $\sigma_{1}$ will present the same temperature dependence as in Fig. 10.

swever, a more ambitious objective is to really predict the effect of temperature (and rain rate) on the ductile fracture properties of the material. For this, the inverse effect rain rate on the flow curve $R(p)$ is introduced (see the Appendix)

$$
R(p, \dot{p})=R(p)+h(\dot{p})
$$

Im the smooth and notched tension tests performed at $300^{\circ} \mathrm{C}$ and at various strain rates eats $\mathrm{B}$ and $\mathrm{D}$, including those in Ref 12 , the following relationship can be used as a ipproximation

$$
R(p, \dot{p})=R_{\dot{p}_{0}}(p)-K p \ln \left(\frac{\dot{p}}{\dot{p}_{0}}\right)
$$

e $K=50 \mathrm{MPa}$, and $\dot{p}_{0}=10^{-3} \mathrm{~s}^{-1}$ is the reference strain rate of the characterization . In the step-by-step elastoplastic numerical calculation, the equivalent strain rate $\dot{p}$

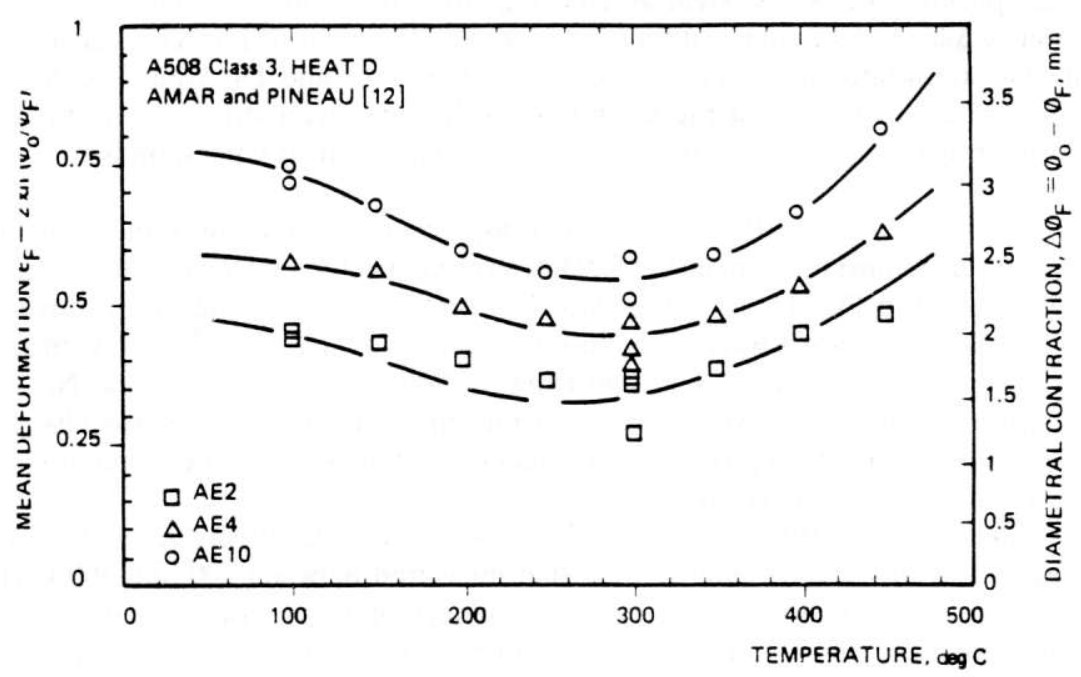

FIG. 10-Temperature dependence of fracture initiation in notched tension specimens. 


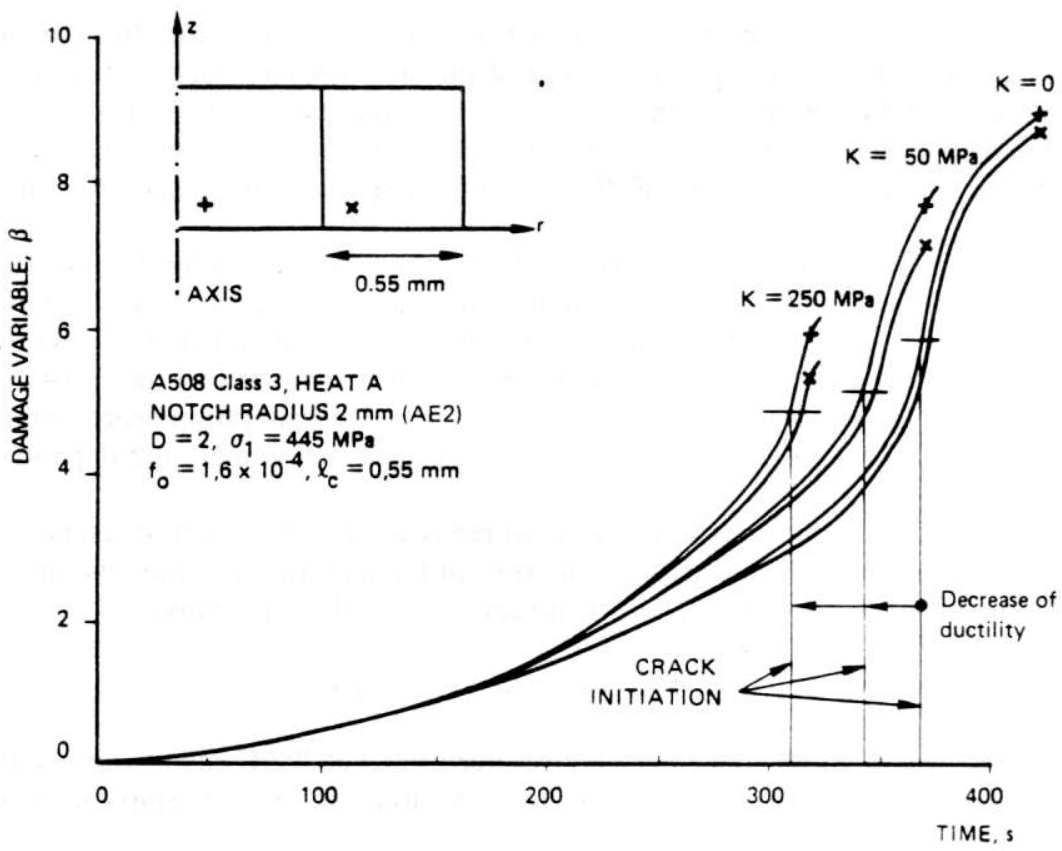

FIG. 11-Inverse strain rate effect on damage in a notched tension specimen.

calculated at the preceding step is used in Eq 4 to determine the hardening curve of the current step.

In the first attempt to model the strain aging effect, the reference calculation is that of an AE2 specimen of A508, Heat A (see Fig. 6). The diametral contraction at fracture initiation is $\Delta \phi_{F}=1.62 \mathrm{~mm}$, without strain aging; $K=0$ in Eq 4. Two calculations with the inverse strain rate effect were performed, with $K=50$ and $250 \mathrm{MPa}$. The displacement rate $\dot{u}_{z}$ imposed at the end of the mesh is such that the mean strain rate in the minimum cross section is $\dot{\epsilon}=-2 \dot{\phi} / \phi=10^{-3} \mathrm{~s}^{-1}$; at the larger strains it corresponds to $\dot{u}_{z}=1.4 \times$ $10^{-3} \mathrm{~mm} / \mathrm{s}$.

As $h(\dot{p})=0$ for $\dot{p}=10^{-3} \mathrm{~s}^{-1}$, the load-displacement curves resulting from the three calculations are almost indistinguishable. But, as was expected, the local values of the damage variable, plotted in Fig. 11, are progressively more important in the case of inverse strain rate effect, and a forward fracture initiation is obtained: $\Delta \phi_{F}=1.58 \mathrm{~mm}$ (time $=343 \mathrm{~s}$ ) for $K=50 \mathrm{MPa}$, and $\Delta \phi_{F}=1.47 \mathrm{~mm}($ time $=309 \mathrm{~s})$ for $K=250 \mathrm{MPa}$. Note that the same parameter, $\sigma_{1}=445 \mathrm{MPa}$, is used in the three calculations. As was also expected, the inverse strain rate effect gives smaller values of the damage variable at fracture initiation, that is, smaller critical cavity growths.

But even with the artificially enlarged inverse strain rate effect $(K=250 \mathrm{MPa})$, the decrease in ductility $\Delta \phi_{F}$ is smaller than that measured between 100 and $300^{\circ} \mathrm{C}$ (Fig. 10). So, before a real prediction of the temperature effect on ductile fracture can be used, more experimental work on material characterization at $300^{\circ} \mathrm{C}$ and a more refined numerical algorithm for the inverse strain rate effect are needed. 


\section{Application to Cracked Structures}

The preceding sections were devoted to material characterization and to the prediction of material properties as they are affected by various factors. The transferring of the characterization data to structural situations has still to be illustrated-that is to say, to what extent has the model, calibrated with notched tension tests, the ability to simulate the behavior of a cracked structure? In the present application, the structures are simple circumferentially fatigue precracked tension specimens (see Figs. 12 and 13); this geometry was chosen because it can be analyzed with a two-dimensional calculation. Other applications to different geometries and thermomechanical loadings are in the making and cannot be reported in this short paper.

Two different specimen sizes were tested at $100^{\circ} \mathrm{C}$; the outer diameters were $\phi=30$ and $50 \mathrm{~mm}$. All specimens were made of A508 Class 3 steel, Heat A. The radii of the uncracked sections after fatigue precracking were $r_{0}=8.3 \pm 0.2 \mathrm{~mm}(\phi=30 \mathrm{~mm})$ and $r_{0}=13.8 \pm$ $0.2 \mathrm{~mm}(\phi=50 \mathrm{~mm})$; the initial crack length was $a_{0}=\phi / 2-r_{0}$. The elongation was measured on gage lengths $L_{0}=78.4 \mathrm{~mm}(\phi=30 \mathrm{~mm})$ and $L_{0}=130.6 \mathrm{~mm}(\phi=50 \mathrm{~mm})$. The interrupted test method was used: the specimens were loaded up to various elongations, unloaded, heat-tinted, and broken in liquid nitrogen. This allowed a direct measurement of the crack growth $\Delta a$. Note that $\Delta a$ includes the "stretch zone" due to the initial blunting of the crack tip. More details can be found in Ref 13. Each specimen gives only one point (plotted in Fig. 12 or Fig. 13) of the experimental crack resistance curve-the loading parameter versus crack growth. The loading parameter is the elongation and not the

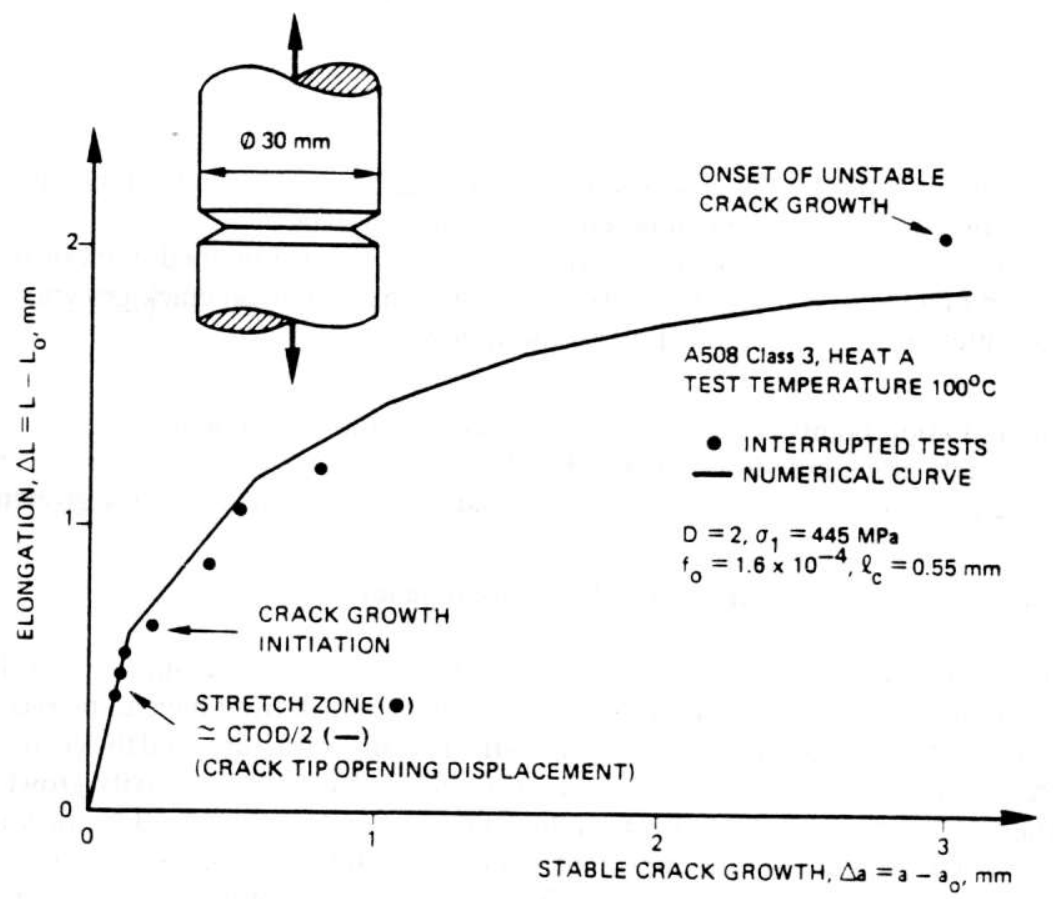
$\mathrm{mm}$. 


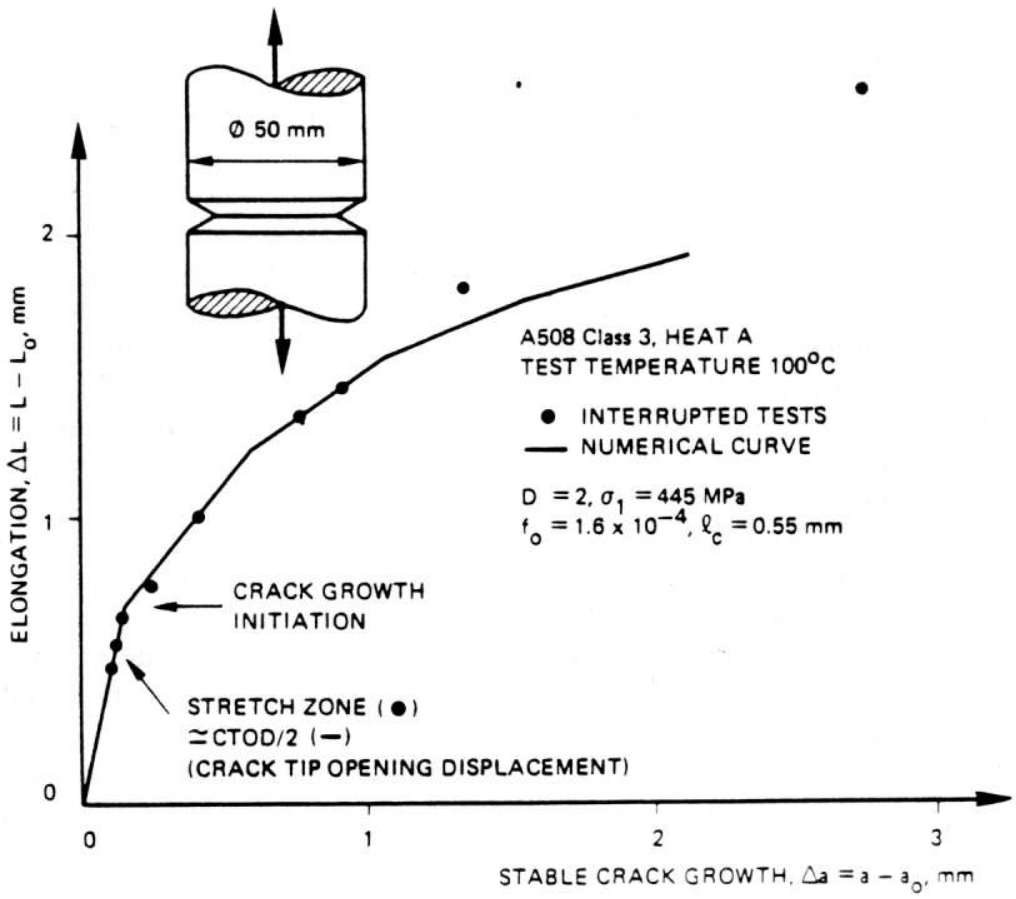

FIG. 13-Crack resistance curve of a circumferentially precracked tension specimen $(\phi 50$ $\mathrm{mm})$.

$J$-integral, usually plotted in a crack resistance diagram. The elongation data are the direct experimental data to be compared with calculated data.

Just as in the notched specimens, the stable crack growth is obtained numerically by the propagation of a highly damaged zone (see Fig. 14). The numerical crack growth resistance curves are plotted in Figs. 12 and 13 with the following results:

1. The initiation points coincide almost exactly with the experimental ones.

2. The stable crack growth is predicted with very good accuracy up to $\Delta a \simeq 1 \mathrm{~mm}$.

3. For $\Delta a>1 \mathrm{~mm}$, the calculation overestimates the experimental crack growth.

At least two factors can explain this latter discrepancy:

1. In real three-dimensional ductile crack growth, the crack surface, initially flat, becomes more and more distorted. The different planes along the crack front have to be reconnected by shear bands for the crack to continue its growth. This shear fracture, and the corresponding additional energy, are not taken into account in the model, based on cavity growth.

2. There is a statistical effect, in which the volume of material involved in crack initiation and early crack growth is more important than that involved in subsequent crack growth. Thus, the probability of having large inclusions in that volume of material is lower for large crack growth, and a statistically lower value of $f_{0}$ should be used. Note that both statistical 


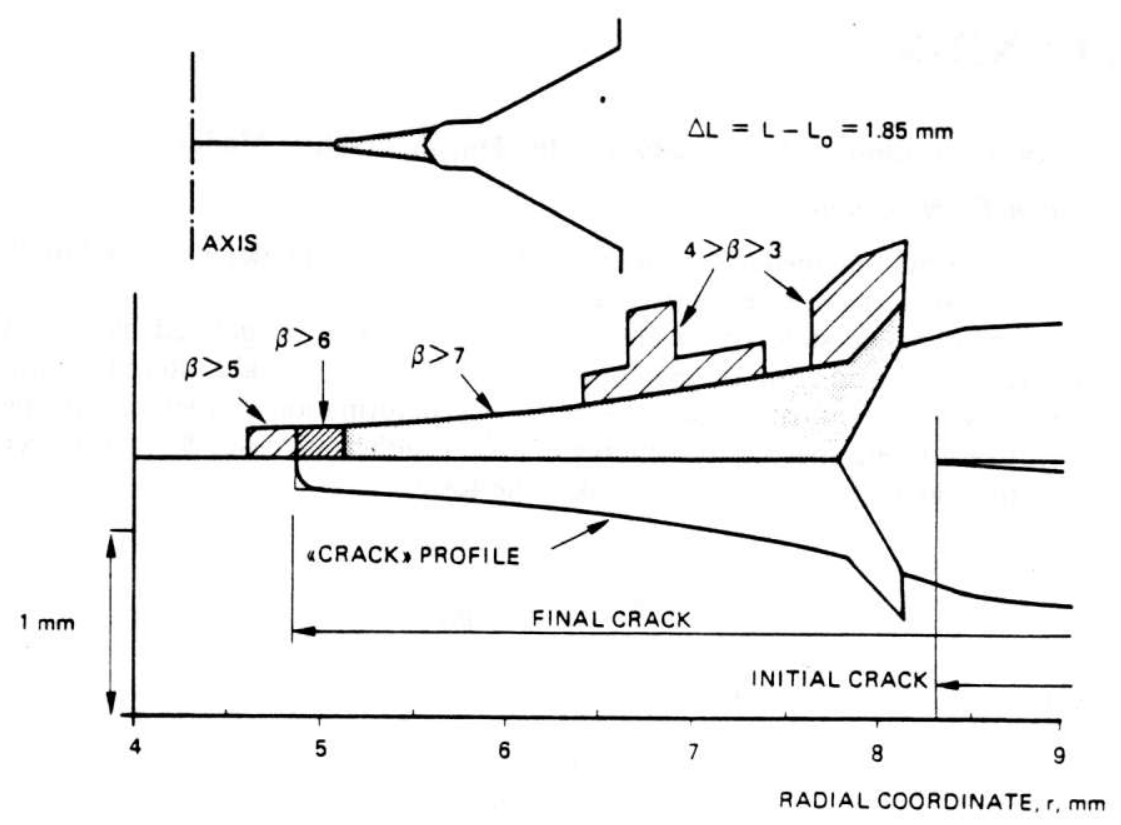

FIG. 14-Damaged zones in a circumferentially precracked tension specimen $(\phi 30 \mathrm{~mm})$.

and three-dimensional effects could be taken into account with an artificially lower value of $f_{0}$. However, as a whole, the numerical simulation of crack initiation and growth can be considered of good accuracy for industrial applications.

\section{Conclusions}

A new methodology for ductile fracture analysis, based on a local approach to fracture and damage mechanics, has been applied to the characterization of structural steels: a Ni$\mathrm{Cr}-\mathrm{Mo}$ rotor steel, an austenitic weld, and several heats of A508, Class 3 steel. The complete characterization can be performed with simple measurements and tests on notched tension specimens.

An effective prediction of the effects of inclusion content and temperature on ductile fracture has been attempted, with favorable results for the inclusion content effect, through a specific parameter of the model. For the temperature effect, a more refined numerical modeling of the inverse strain rate effect on the stress-strain curve should be the subject of future work.

The transferring of material characterization data to cracked structures is direct, and the numerical simulation of crack initiation and growth in circumferentially cracked tension specimens is in good agreement with the experimental results. Additional applications in the case of thermomechanical loadings are in the making, with favorable preliminary results. 


\section{APPENDIX}

\section{Theoretical Derivation of the Equations of the Ductile Fracture Model}

\section{Continuum Thermodynamics}

Only the information needed for the comprehension of the model is recalled in this short section. For more details, refer in particular to Ref 14 .

The thermodynamic state of an element of material can be defined by "observable" variables (elastic deformation $\epsilon^{e}$, temperature $T$, and so forth) and "internal" variables $\alpha$, which reflect various dissipative mechanisms (plastic deformation (hardening), damage, and so forth). Considering specific free energy $\phi$ in the isothermal case, $\phi=\phi\left(\epsilon^{e}, \alpha\right)$, it can be shown that dissipated power $\Phi>0$ takes the form

$$
\Phi=\frac{\sigma}{\rho} \dot{\epsilon}^{p}-\frac{\partial \phi}{\partial \alpha} \dot{\alpha}
$$

and that

$$
\frac{\sigma}{\rho}=\frac{\partial \phi}{\partial \epsilon^{e}}
$$

where $\rho$ is the mass per unit of volume. Entering the "forces" $A$ associated with $\dot{\alpha}$

$$
A=-\frac{\partial \phi}{\partial \alpha}
$$

Equation 5 is thus written in the form

$$
\Phi=\Sigma Y_{i} X_{i}
$$

with $X=\left(\dot{\epsilon}^{p}, \dot{\alpha}\right)$ and $Y=(\sigma / \rho, A)$. The definition of constitutive relations consists of linking the $\mathrm{X}$ to the $\mathrm{Y}$.

In the standard model, viscoplastic case, a convex viscoplastic potential $\Omega(Y)$, and criterion of plasticity, $F=0$, are entered, so that $X=\lambda \partial F / \partial Y$; that is

$$
\begin{gathered}
\dot{\boldsymbol{\epsilon}}^{p}=\lambda \frac{\partial F}{\partial\left(\frac{\sigma}{\rho}\right)} \\
\dot{\alpha}=\lambda \frac{\partial F}{\partial A}
\end{gathered}
$$

The first relationship (Eq 8) is the classical normality rule. In the standard model, the behavior of the material is entirely defined by the choice of the two potentials, $\phi\left(\epsilon^{e}, \alpha\right)$ and $F(\sigma / \rho, A)$ or $\Omega(\sigma / \rho, A)$. 
In the application to ductile fracture, the internal variables are divided into:

(a) a hardening variable marked $p$ and

(b) a damage variable marked $\beta$.

\section{Derivation of the Model}

Having defined the purpose of the model as the plastic deformation and ductile fracture damage of metals, and its scope as the continuum damage mechanics, in the final analysis the model is based on two concepts only:

(a) the "principle of simplicity" (choice of the simplest formulation in a given theoretical framework) and

(b) the standard model (Hypothesis H1).

The application of the principle of simplicity leads to the hypotheses set out below:

Hypothesis $\mathrm{H} 2$ - The internal hardening and damage variables $p$ and $\beta$ are scalar (hypothesis of isotropy), and the "forces" associated with $p$ and $\beta$ are denoted $P$ and $B$, respectively.

Hypothesis $\mathrm{H} 3$ - The thermodynamic potential $\phi\left(\epsilon^{e}, p, \beta\right)$ is of the form

$$
\phi=\phi_{e}\left(\epsilon^{e}\right)+\phi_{p}(p)+\phi_{\beta}(\beta)
$$

which, according to Eq 7, simply yields $P=P(p)$ and $B=B(\beta)$. The elastic constitutive relationships are assumed to be linear, hence

$$
\phi_{e}=\epsilon^{e} L \epsilon^{e} / 2
$$

according to Eq 6. $L$ is the matrix of elastic moduli.

Hypothesis $\mathrm{H} 4$ - The plastic potential $F(\tilde{\sigma}, P, B)$, in which $\tilde{\sigma}=\sigma / \rho$, is of the form

$$
F=F_{1}\left(\tilde{\sigma}_{\text {eq }}, P\right)+F_{2}\left(\tilde{\sigma}_{m}, B\right)
$$

where

$$
\begin{gathered}
F_{1}=\tilde{\sigma}_{\mathrm{eq}}+P(p)(\text { von Mises yield criterion) } \\
F_{2}=B(\beta) g\left(\tilde{\sigma}_{m}\right)
\end{gathered}
$$

Hypothesis $\mathrm{H} 4$ calls for some comment.

1. F only depends on the first two invariants of the stress tensor. The specific nature of the model (ductile fracture) appears through the first invariant $\sigma_{m}$.

2. The additive form (Eq 9), only, yields simple constitutive relationships in light of Eq 8 of the standard model. Equation 10 is also the simplest possible.

3. $\tilde{\sigma}=\sigma / \rho$ and not $\sigma$ enters the plastic potential, in light of the expression of dissipated power (Eq 5). In classical plasticity, the hypothesis of incompressibility makes it possible to neglect the variations of $\rho$, but this hypothesis is no longer acceptable for modeling ductile fracture. In the present model, in the absence of damage $(\beta=$ constant $)$, we have $\rho=$ 
constant, except for elastic deformations; in the opposite case ( $\beta$ variable), one can write $\rho=\rho(\beta)$ as a first approximation.

The consequences of hypotheses $\mathrm{H} 1$ to $\mathrm{H} 4$ are examined below.

Neglecting the variations in volume due to elastic deformations, the mass preservation law can be written

$$
\dot{\rho}+3 \rho \dot{\epsilon}_{m}^{p}=0
$$

However

$$
\dot{\rho}=\rho^{\prime}(\beta) \dot{\beta}
$$

and the relations in Eqs 8 yield

$$
\begin{gathered}
3 \dot{\boldsymbol{\epsilon}}_{m}^{p}=\lambda B(\beta) g^{\prime}\left(\tilde{\boldsymbol{\sigma}}_{m}\right) \\
\dot{\beta}=\lambda g\left(\tilde{\boldsymbol{\sigma}}_{m}\right)
\end{gathered}
$$

Consequently

$$
\frac{g^{\prime}\left(\tilde{\sigma}_{m}\right)}{g\left(\tilde{\sigma}_{m}\right)}=-\frac{\rho^{\prime}(\beta)}{B(\beta) \rho(\beta)}
$$

The two members of this equation are functions of distinct variables, $\tilde{\sigma}_{m}$ and $\beta$; they are therefore equal to a constant, the dimension of which is the reciprocal of a stress and which is called $1 / \sigma_{1}$. The integration of $g^{\prime} / g=1 / \sigma_{1}$ leads to

$$
g\left(\tilde{\sigma}_{m}\right)=D \exp \left(\frac{\tilde{\sigma}_{m}}{\sigma_{1}}\right)
$$

in which $D$ is the constant of integration. Here, $\sigma_{1}$ and $D$ are the first two parameters to appear in the model.

In light of Eq 11, the plastic potential is written as in Eq 2

$$
F=\frac{\sigma_{e q}}{\rho}-R(p)+B(\beta) D \exp \left(\frac{\sigma_{m}}{\rho \sigma_{1}}\right)
$$

in which $R(p)=-P(p)$ is the hardening curve of the material. The relations in Eqs 8 of the standard model finally lead to

$$
\begin{gathered}
\dot{e}_{i j}^{p}=\dot{p} \frac{3 s_{i j}}{2 \sigma_{\text {eq }}} \\
\dot{\boldsymbol{\epsilon}}_{m}^{p}=\dot{p} \frac{B(\beta)}{3 \sigma_{1}} D \exp \left(\frac{\sigma_{m}}{\rho \sigma_{1}}\right) \\
\dot{p}=\lambda \\
\dot{\beta}=\dot{p} D \exp \left(\frac{\sigma_{m}}{\rho \sigma_{1}}\right)
\end{gathered}
$$


where

and

$$
\dot{\boldsymbol{\epsilon}}_{m}^{p}=\frac{\dot{\boldsymbol{\epsilon}}_{i i}^{p}}{3}
$$

$$
\begin{aligned}
& s_{i j}=\sigma_{i j}-\sigma_{m} \delta_{i j} \\
& \dot{e}_{i j}^{p}=\dot{\epsilon}_{i j}^{p}-\dot{\epsilon}_{m}^{p} \delta_{i j}
\end{aligned}
$$

are the deviators of $\sigma$ and $\dot{\boldsymbol{\epsilon}}^{p}$, respectively. Computation of $\dot{\boldsymbol{\epsilon}}_{\mathrm{cq}}^{p}$ yields $\dot{\boldsymbol{\epsilon}}_{\mathrm{eq}}^{p}=\dot{p}$; the internal hardening variable thus identifies with the cumulated equivalent plastic deformation.

The function $B(\beta)$ remains to be specified. Considering cavities of any shape with an average volume $v$ in an incompressible matrix, it is easy to show that the volume fraction of cavities $f$ is such that

$$
\frac{\dot{f}}{f(1-f)}=\frac{\dot{v}}{v}
$$

Moreover, the mass per unit of volume is given by

$$
\frac{\rho}{\rho_{0}}=\frac{1-f}{1-f_{0}}
$$

To simplify the notation, $\rho$ is defined as the relative density obtained by dividing the density of the damaged material by that of the material in its initial state: the initial value of $\rho$ is therefore $\rho_{0}=1$. Equations 14 and 15 yield

$$
\frac{\dot{v}}{v}=\frac{-\rho^{\prime} \dot{\beta}}{\rho\left(1-\rho+\rho f_{0}\right)}
$$

As a result of Eq 13 and $\dot{p}=\dot{\epsilon}_{\mathrm{eq}}^{p}$

$$
\frac{\dot{v}}{v}=\left[-\frac{\rho^{\prime}}{\rho\left(1-\rho+\rho f_{0}\right)}\right] D \dot{\boldsymbol{\epsilon}}_{\mathrm{eq}}^{p} \exp \left(\frac{\sigma_{m}}{\rho \sigma_{1}}\right)
$$

There is a striking analogy with the equation of Rice and Tracey (Eq 1): it should be recalled that the exponential form is not a hypothesis in itself but that it flows from Hypotheses $\mathrm{H} 1$ to $\mathrm{H} 4$. Through a new and last application of the principle of simplicity, it is assumed (Hypothesis H5) that Eq 16 reduces to

$$
\frac{\dot{v}}{v}=D \dot{\boldsymbol{\epsilon}}_{\mathrm{eq}}^{p} \exp \left(\frac{\sigma_{m}}{\rho \sigma_{1}}\right)
$$

that is, to the Rice and Tracey equation if $D=3 \times 0.283, \rho=1$, and $\sigma_{1}=2 \sigma_{\text {eq }} / 3=$ $2 \sigma_{0} / 3$ in the nonhardening case. (Note that $D=2$ was used in this paper, in agreement with cavity growth measurements; the Rice and Tracey coefficient 0.283 is recognized to be too small [4].) Hypothesis H5 implies

$$
\rho(\beta)=\frac{1}{1-f_{0}+f_{0} \exp \beta}
$$


and given that $-\rho^{\prime} / \rho B=1 / \sigma_{1}$, Eq 17 yields Eq 3

$$
B(\beta)=\frac{\sigma_{1} f_{0} \exp \beta}{1-f_{0}+f_{0} \exp \beta}
$$

A third parameter, the initial volume fraction of cavities $f_{0}$, is thus added to $\sigma_{1}$ and $D$. The use of Eq 17 is optional; in a numerical computation, $\rho$ can be deduced directly from the field of displacements.

The model is thus fully defined with the only guidance being systematic selection of the simplest formulation.

\section{Viscoplastic Case}

Again, according to the principle of simplicity, the viscoplastic potential is in the form $\Omega(Y)=\Omega(F(Y))$, with $F(\tilde{\sigma}, P, B) \neq 0$ in the viscoplastic case. As

$$
\dot{p}=\frac{\partial \Omega}{\partial P}=\frac{d \Omega}{d F} \cdot \frac{\partial F}{\partial P}=\frac{d \Omega}{d F}
$$

the other components of $X=\left(\dot{\epsilon}^{p}, \dot{p}, \dot{\beta}\right)$ are given by $X=\dot{p} \partial F / \partial Y$, exactly as in the plastic case (Eqs 12 and 13), in which also $\dot{p}=\dot{\boldsymbol{\epsilon}}_{\mathrm{eq}}^{p}$.

The difference is that $F=h(\dot{p})$, instead of $F=0$ in the plastic case, where $h$ is the reciprocal function of $d \Omega / d F . F=h(\dot{p})$ gives

$$
\tilde{\sigma}_{\text {eq }}-R(p)-h(\dot{p})+B(\beta) D \exp \left(\frac{\tilde{\sigma}_{m}}{\sigma_{1}}\right)=0
$$

One interpretation of the viscoplastic model is to handle it numerically like a plastic model, but with a hardening curve depending on the strain rate

$$
R(p, \dot{p})=R(p)+h(\dot{p})
$$

\section{References}

[1] Devaux, J. C., Saillard, P., and Pellissier-Tanon, A., "Elastic-Plastic Assessment of a Cladded Pressurized-Water-Reactor Vessel Strength Since Occurrence of a Postulated Underclad Crack During Manufacturing," this publication, pp. 454-469.

[2] D'Escatha, Y. and Devaux, J. C. in Elastic-Plastic Fracture, ASTM STP 668, American Society for Testing and Materials, Philadelphia, 1979, pp. 229-248.

[3] Rousselier, G. in Three Dimensional Constitutive Relations and Ductile Fracture, North Holland Publishing Company, Amsterdam, 1981, pp. 331-355.

[4] Rousselier, G. in Proceedings, International Seminar on Local Approach of Fracture, Electricité de France/Etude des Matériaux, Les Renardières, 77250 Moret-sur-Loing, France, 3-5 June 1986, pp. 257-284.

[5] Franklin, A. G., Journal of the Iron Steel Institute, 1969, p. 181.

[6] Rousselier, G. in Proceedings, Specialists Meeting on Elastoplastic Fracture Mechanics, OECD NEA-CSNI Report 32, OECD Nuclear Energy Agency, Paris, France, 1978, pp. 14.1-14.15.

[7] Devaux, J. C., Mottet, G., Balladon, P., and Pellissier-Tanon, A. in Proceedings, International Seminar on Local Approach of Fracture, Electricité de France/Etude des Matériaux, Les Renardières, 77250 Moret-sur-Loing, France, 3-5 June 1986, pp. 321-333.

[8] Batisse, R., Bethmont, M., Devesa, G., and Rousselier, G. in Proceedings, International Seminar on Local Approach of Fracture, Electricité de France/Etude des Matériaux, Les Renardières, 77250 Moret-sur-Loing, France, 3-5 June 1986, pp. 285-298. 
[9] Kussmaul, K., Sauter, A., Nguyen-Huy, T., and Feyhl, D. in Proceedings, Eighth International Conference on Structural Mechanics in Reactor Technology, Bruxelles, Belgium, August 1985, pp. $169-175$.

[10] Ostensson, B. and Westin, R., "The Influence of Temperature and Strain Rate on Fracture Toughness of A 533 B Pressure Vessel Steel Plate Material," AB Atomenergi Report S-536, Studsvik, Nykoping, Sweden, 1976.

[11] Sandberg, O. in Proceedings, Eighth International Conference on Structural Mechanics in Reactor Technology, Bruxelles, Belgium, August 1985, F2 1/2, pp. 409-416.

[12] Amar, E. and Pineau A., Engineering Fracture Mechanics, Vol. 22, No. 6, 1985, pp. 1061-1071.

[13] Devaux, J. C., Rousselier, G., Mudry, F., and Pineau, A., Engineering Fracture Mechanics, Vol. 21, No. 2, 1985, pp. 273-283.

[14] Germain, P., Nguyen, Q. S., and Suquet, P., Journal of Applied Mechanics, Vol. 50, December 1983, pp. 1010-1020. 